\title{
Joint epistemic engineering: The neglected process in human communication
}

\author{
Arjen Stolk, ${ }^{1,2}$ Jana Bašnáková, ${ }^{2}$ Ivan Toni ${ }^{2}$ \\ ${ }^{1}$ Psychological and Brain Sciences, Dartmouth College, Hanover, NH, USA \\ ${ }^{2}$ Donders Institute for Brain, Cognition, and Behaviour, Radboud University, Nijmegen, the Netherlands
}

\begin{abstract}
How do inherently asymmetric interlocutors rapidly arrive at mutually understood mental constructs from ambiguous signals that are referentially contingent on the current communicative circumstances? This question is at the core of the neurosemiotic agenda. This chapter introduces joint epistemic engineering, an account of cognitive and neurobiological mechanisms supporting the construal of signs and meanings during interactive human communication. The basic insight is that interlocutors use multi-layered signals as a tool to simultaneously probe, align, and shape their conceptual structures of the interaction, constructing a shared and situated source of interpretational constraints on the meaning of those signals. We review recent empirical observations on the neural implementation of joint epistemic engineering, and we show the potential of this neurosemiotic account for understanding disorders of human communication, as seen, for example, in autism spectrum disorder.
\end{abstract}

Keywords: social interaction, mutual understanding, conceptual alignment, brain, autism. 
There is not much dependence to be placed upon these Constructions that we put upon Signs and Words, which we understand but very little of, \& at best can only give a probable Guess at their Meaning.

David Samwell, ship surgeon on James Cook’s HMS Discovery, Hawaii, 1779

\section{Introduction}

There is virtually no limit to what human minds can conceive beyond the physical world. Those mental activities might appear inconsequential or delusional. Yet, when people collectively agree on mental constructs, those cultural innovations can change reality, as when we attribute value to intrinsically worthless banknotes. Ultimately, those cultural innovations are rooted in our communicative abilities (Levinson, 2006; Misyak et al., 2014). How human minds communicate and converge on mental constructs, however, remains a largely open question. By focusing on both cognitive and neurobiological mechanisms of meaning construal during interactive communication, neurosemiotics offers a promising approach to tackle this hard question (García et al., 2020). More precisely, this chapter adopts a neurosemiotic focus on the mechanistic unfolding of the processes that create relations between signs and referents.

A core assumption in language and communication research holds that interlocutors understand one another because they have approximated the same conceptual structures 
through lifelong exposure to publicly available signals and a common mental lexicon storing information regarding their meanings (Gaskell, 2007). On this assumption, human communication is largely understood when we know how conventional signals, such as words, gestures, and facial expressions, are encoded and decoded by individuals. However, that traditional view ignores two key features of human communication. First, as repeatedly emphasized by semioticians, there are many-to-many mappings between a signal's form and meaning (Kull, 2020). Given that no two people share the same experience and expertise, interlocutors rarely, if ever, share the same default set of candidate meanings when interpreting a signal (Clark \& Marshall, 1981; Lewis, 1969). Second, the meaning of any signal is negotiable and ultimately depends on the contingently unfolding interaction between interlocutors (Brennan et al., 2010; Tomasello et al., 2005). This is seen in how everyday interaction consists of ambiguous words and behaviors that can only be discerned by individuals who know their context of use in an ongoing interaction. Notwithstanding helpful regularities in word order and dialogic turns (subject-verb-object structures, question-answer relationships, social scripts, and other stereotyped dependencies), everyday interaction is also astonishing in its flexibility. An utterance can constitute a response to another verbal or nonverbal utterance that occurred at any point in time along the interaction's trajectory, thus irrespective of word order, turn sequence, or even communication modality. These fleeting conceptual dependencies between utterances cannot easily be analyzed outside the communicative contexts in which they are embedded, as state-of-the-art artificial agents, such as Alexa or Siri, try to do.

Building on previous suggestions (Clark, 1996; Grice, 1957; Peirce, 1931), we argue that a common language and its statistics do not explain how people in dialogue overcome fundamental communicative obstacles. The reason is that signals do not have stereotyped and 
publicly invariant consequences. The real challenge is to specify how people define a context rich enough to afford a signal a fair chance at being interpreted in the way it is intended at the

moment of delivery, yet flexible enough to be continuously adjusted to the goals and needs of the conversation. We review recent work in neurosemiotics that has begun to shift focus from the context-free coding-decoding of signals to the construal of meaning within genuinely interactive contexts. Extending early observations of a shared conceptual space underlying human communication (Stolk et al., 2016; Toni \& Stolk, 2019), here we introduce "joint epistemic engineering," an account of how interlocutors can understand each other in everyday conversation as well as in situations when meaning needs to be construed from scratch.

The basic insight is that a large portion of a signal's context of use is constructed on-the-fly by members of the interaction. In what follows, we illustrate how interlocutors use multilayered communicative signals as a tool to simultaneously probe, align, and shape their conceptual structures during social interaction, providing a shared and situated source of interpretational constraints. By embedding signals in the conceptual space generated through this engineering process, interlocutors rapidly zoom in on relevant features of a signal and coordinate both current and new reference points in their frame of reference of the interaction.

\section{From pre-Darwinian types to contingently shared tokens}

By way of analogy, considering human communication as a signal coding-decoding problem is a fallacy reminiscent of the focus that pre-Darwinian natural sciences had on biological types. In that pre-Darwinian view, time-invariant species were the fundamental units of biological organization, worthy of investigation because of their presumed immutability and 
optimality (Mayr, 1999). Arguably, one of Darwin's main conceptual innovations was to shift the focus of inquiry from biological types to tokens; from ideal species to individual organisms that might or might not pass through the filter of natural selection. In Darwin's framework, each accident of history became informative, showing how a biological replicator adapts to the current environmental circumstances in light of past adaptations. In contrast, the focus of linguistics has largely remained anchored on signal types, as seen, for instance, in the academic space devoted to investigating regularities in word order and turn-taking patterns (Gaskell, 2007; Sacks et al., 1974). Deviations from those regularities, frequent in real-life dialogue (Stivers, 2010), have been largely ignored as stochastic phenomena, rather than accidents of interactional history showing how interlocutors create and adapt to the current communicative circumstances.

Linguistic types are thought to offer optimal solutions to the problem of linking a set of signals to their meanings, with a pre-defined syntax combining signals compositionally to form a larger meaning (Gibson et al., 2019). Those solutions are optimal insofar as human communication is framed as an information-theoretic problem - i.e., the problem of reducing uncertainty over the identity of a signal given the set of possible signals and referents (Shannon, 1948). Information-theoretic frameworks assume that linguistic signals have meanings shared with other members of our linguistic community, up to the point where those meanings are properties of those signals. However, while there are obvious surface regularities in the signals we use, they do not map reliably onto regularities in the content of the signals - e.g., the sound /dog/ onto its possible referents. Those referents are highdimensional concepts (e.g., an individual pet, a zoological category, a human villain) that require similar contextual constraints to afford similar interpretations across communicators. As a painter needs to decide which hue to contrive from her palette for achieving a fair 
chance the viewer will perceive a red apple in the context of that particular canvas (Conway, 2012), so a communicator needs to decide how to contrive a signal that will be interpreted as intended in the current communicative context, even when the signal might seem highly conventional.

Building on these considerations, this chapter raises two main conceptual issues, in line with a large body of work on experimental semiotics and pragmatics (Galantucci \& Garrod, 2011; Noveck \& Reboul, 2008). First, we advocate a shift from pre-Darwinian type-centered thinking, where signals have stereotyped and publicly invariant consequences, toward studying the actual tokens used during communicative interactions. Type-centered frameworks have been instrumental in extracting linguistic regularities from idealized material, like individual agents processing isolated sentences. Here, we argue that, when signals are considered in their natural embedding of contingently unfolding social interaction, then human communication should be thought of as joint control of a shared conceptual space, rather than signal coding-decoding. Second, we emphasize that interlocutors' overlaps in signal-referent mappings and communicative contexts are a product of communication, not a default background. We explore a way these interpersonal disparities can be resolved on the fly, namely the suggestion that a communicative signal contains a multiplicity of semiotic functions. The basic insight is that a communicative token, besides being deployed to reduce uncertainty over the identity of its referent - a Shannon-signal (Shannon, 1948) -, is also meant to ostensively mark its own communicative value - a Grice-signal (Grice, 1957) - and to offer cues for aligning the space of possible interpretations across interlocutors - a Peircesignal (Peirce, 1931).

\section{What is in a signal?}




\section{Shannon-signals}

In the original transmission-capacity view of communication (Shannon, 1948), there is no mechanism for assigning meaning to a signal. A signal conveys information to a receiver when it reduces the receiver's uncertainty over the identity of that signal given the set of possible signals and referents. It is assumed that both sender and receiver share a pre-existing set of signal-referent mappings, like two telegraph operators sharing the Morse code. Natural and simulated selection has led to numerous situations where conspecifics can rely on shared signal-referent mappings (Danchin et al., 2004; Kirby et al., 2014). In those situations, a communicator can generate Shannon-signals, since the set of possible signals and the set of signal-referent mappings are already fully shared by senders and receivers. Alarm calls of vervet monkeys, bees' dances, or human behavioral contagion are just a few examples of Shannon-signals. However, this communicative scheme requires repeated rounds of natural selection under consistent pressures to arise. Accordingly, it is not clear how it can account for the referential flexibility of human communication, as seen in the moment-to-moment variability in signal use across and within conversations. It is also not obvious how referential flexibility can be accounted for by ignoring signal-referent mappings altogether - e.g., explanatory frameworks where signal use is determined exclusively by an iterated process of reciprocal influence and resistance between individuals, devoid of any reference (Dawkins \& Krebs, 1978; Rendall \& Owren, 2013).

\section{Grice-signals}

Addressing referential flexibility, Grice initiated an influential perspective on human communication, arguing that it requires interlocutors to have knowledge about one another's intentions and beliefs to disambiguate the intended meaning of a signal (Grice, 1957). A Grice-signal involves a sender producing a signal to (i) induce functional changes in an 
interpreting receiver, (ii) assess that the signal has been recognized as to induce those changes, and (iii) ultimately give rise to functional changes that incorporate consequences of that recognition. As when a driver flashes her lights to an oncoming car, a signal is also meant to ostensively mark its own communicative value, signaling that we not only intend to influence the receiver (through the production of a stimulus), but also want them to recognize that we are acting with such intentions and, ultimately, respond on the basis of recognizing this (Sperber \& Wilson, 1986). In this scheme, interlocutors can go beyond literal meaning as long as their respective speech acts adhere to the accepted purpose or direction of the conversation as well as universal principles of cooperation. As part of the latter, Grice indicates that each individual's contribution ought to be as relevant and informative as required, but not more than required for the current purpose of the exchange. He leaves unspecified, however, how interlocutors determine what quantity of information is appropriate for the current purposes. What is considered concise by one, could be pedantic to another.

\section{Peirce-signals}

Emphasizing interpretation, Peirce's triadic model highlighted a special role for meaningmaking agents in the communication process, adding a third entity to dyadic models of communicative tokens and the mental concepts they kindled. A Peirce-signal (or sign, in Peircean terms) is what it is interpreted to be, as when clouds signify rain to some, and ruins stand for ancient civilization to others. The triadic representation of a referent entails that a Peirce-signal can take any form, and that nothing is a communicative token unless it is interpreted as such (Peirce, 1931). Peirce further distinguished three primary signal types: icons, indices, and symbols. The former two bear a natural relationship with the object they represent, either through perceptual resemblance (icons, e.g., two fingers up for the number 
two) or through physical or causal connection (indices, e.g., two fingers for $\mathrm{V}$ for victory). For their part, symbols signify an object only via an arbitrary rule imposed by a community (e.g., two fingers up conventionally standing for peace). The problem with this tripartite scheme, as is often the case with type-centered thinking, is that a closer look at everyday interactions frequently yields exceptions to the rule, in this instance that utterances can be composites of two or more signal types. For example, uttering "I caught a fish this long" while holding hands a certain distance apart both symbolizes and denotes the length of a caught fish (Clark, 1997). Peirce was aware of this obvious issue, considering the types not mutually exclusive. More generally, however, until we understand how people integrate or otherwise deal with different signal types, any type-centered framework of human communication remains fundamentally limited (Deacon, 2018). Or as the ship surgeon who sailed with James Cook noted in 1779, there is not much dependence to be placed on signal types: they only give a probable guess at a signal's meaning, raising the fundamental issue of understanding how people end up making similar guesses during communicative interactions.

\section{Fixed-code fallacy}

Overall, the multiplicity of functions and referents of a signal argue against the "fixed-code" view that has percolated into modern perspectives of language and communication (Harris, 1998). In these contemporary yet fundamentally pre-Darwinian perspectives, words have universal referents within a linguistic community and encode semantic constructs of thought. We argue that this fixed-code view of language use is an illusion. As we tend to think of language as being lawfully constituted by phonemes, words, and sentences, when in fact those elements are regularized and approximate low-dimensional projections from highdimensional spoken utterances (Elman, 2004; Port, 2010); so we tend to think of communication as being constituted by signals exchanged between interlocutors, when in fact 
those signals are approximate low-dimensional behavioral projections from high-dimensional conceptual spaces in the interlocutors' minds. Put differently, communication requires a degree of conceptual overlap across interlocutors to afford comparable and contextually appropriate interpretations of a candidate behavior, inverting it into partially overlapping concepts (Veissière et al., 2019; Warglien \& Gärdenfors, 2015). This situated inversion would allow those interlocutors to resolve the multiple ambiguities that permeate almost every human interaction, such as distinguishing between a reflexive and an embarrassed cough, or between /dog/ as a pet and /dog/ as a villain. These considerations on the contextdependency of a signal-referent trajectory beg the question of how interlocutors determine what counts as context, a fundamental yet largely overlooked issue we will turn to next.

\section{What counts as context?}

\section{Disambiguation}

When context is brought into models of human communication, it is to arbitrate among the possible interpretations of a signal, as when adjudicating between the meanings of "financial institution" and "riverside" for the sound /bæøk/. This view acknowledges the ambiguities intrinsic in many words. However, there are several problematic assumptions behind the notion that these ambiguities can be fully resolved through contextual factors biasing interpretation toward an intended referent. First, given that no two people share exactly the same experience and expertise, the fact that they are using the same expression does not guarantee perfect symmetry in their conceptual knowledge. When a mechanic talks about a car, she thinks in terms of "brake horsepower" and "electronic stability control," a representation only marginally overlapping with how her customer conceptualizes the same vehicle. Even if expertise in the moment of the conversation was broadly comparable, it seems unlikely that the condition of perfect symmetry between interlocutor can be a priori 
satisfied in the longer term, given how concepts can change over a lifetime, and even within the timespan of a conversation (Carpendale \& Lewis, 2004). It is also unlikely that perfect symmetry is granted by community membership, as no community is sufficiently homogenous to provide full conceptual alignment across interlocutors (Clark \& Marshall, 1981; Lewis, 1969). Second, even assuming perfect symmetry between interlocutors, context is not a clearly defined background, built bottom-up from the sensory data. Signal-referent mappings are plagued by ambiguity; contexts are at least as ambiguous, and harder to define. We do not have sensory receptors for context, e.g., a universal relevance detector (Sperber \& Wilson, 1986). Calling upon context to achieve disambiguation ultimately moves the explanatory problem up a level, without delivering any actual explanation other than ad-hoc heuristics with no generalization power.

\section{Strategic reasoning}

It could be argued that converging on a context is a problem that has already been solved in "game theory," the study of strategic coordination among rational agents. Moving beyond the standard practice of assuming full knowledge of goals and pay-off outcomes built into coordination games (cf. signal-referent mappings), Schelling asked how people could tacitly coordinate given minimal cues (Schelling, 1960). For instance, study participants are given hypothetical problems like meeting with a stranger in New York City on a set date, requiring choosing a time and place. Participants tend to converge on a small set of solutions, a popular answer being noon at Grand Central Station in this particular task. The emergence of these "focal points" has been largely attributed to strategic reasoning, either in the form of modeling and choosing the best response of other players or of an imaginary group leader wanting to maximize group interest. These strategies would arguably afford sufficient flexibility to arrive at a different focal point (e.g., the Statue of Liberty) given another 
relatively well-defined situation (e.g., the stranger is a tourist). However, strategic reasoning presupposes rather than explains how rational agents come to operate on identical levels of granularity in their "New York" representation. For instance, it is unclear whether strategic reasoning would generate effective focal points across agents that do not know whether the coordination challenge is about New York City or New York State. Everyday interlocutors operating in open-ended situations cannot presume overlapping granularity in their contextual representations absent any mutual coordination.

\section{Mutual coordination}

It should follow from the above that "context" is as ambiguous and cognitively construed as the signal being disambiguated. We argue here that it is through the production and interpretation of the Peirce-signals embedded in their communicative history that interlocutors generate a shared context for constraining the possible interpretations of a signal. Besides exchanging signals, communicators jointly determine the detectability and interpretability of those signals. For instance, a communicator could furnish the sound /bæyk/ with a pointed gaze towards the cupboard where both interlocutors know fishing equipment is stored. The mutually known knowledge about the cupboard content, and the physical presence of that piece of furniture, offer a situated source of interpretational constraints for the /bæyk/ sound. The production of an additional Grice-signal (the pointed gaze) poses an interpretational request at the addressee's door, aimed at aligning the interpretational frames of /bæyk/ across the two interlocutors. The addressee might get at the intended referent if she zooms into the focal point provided by the conjunction of the cupboard and her pre-existing shared knowledge with the speaker (the fishing equipment). This trivial example of a daily interaction illustrates the inadequacy of fixed-code approaches to human communication, and the hard inferential labor required to produce and interpret a multi-layered signal. 
Needless to say, the Peirce-signal generated when combining the gaze, the cupboard, and the presumed mutually known knowledge might fail to evoke in the addressee the intended context for the /bæyk/ sound. Under those circumstances, repair operations, including asking for clarification, could prove important for achieving successful communication (Dingemanse et al., 2015). However, repair cannot be the main mechanism supporting successful communication. Calling upon repair to achieve disambiguation ultimately moves the explanatory problem into a secondary channel at least as complex as the original dialogue. In fact, repair procedures seem likely to be computationally more demanding, since the problem of figuring out what is being requested (or what to request) is compounded by the problem of providing a solution to the original ambiguities, and both are rapidly aggravated as time elapses in the turn-taking dynamics. Accordingly, interlocutors often tolerate possible misunderstandings to avoid interrupting a dialogue (Galantucci et al., 2020; Jefferson, 1988).

This tolerance towards possible misunderstanding might not be specifically linked to disagreements, but a core feature of the communication process since interlocutors need to constantly juggle multiple possible interpretational frameworks. In the example above, the addressee might try to embed /bæyk/ into different contexts, e.g., a financial institution, a geographical feature, or a meteorological phenomenon as in a bank of clouds. The addressee might tentatively choose the financial option, but then pivot towards the geographical framework as that initially un-chosen option proves more consistent with the evidence provided by the interlocutor. Tolerance towards potential misunderstanding might result in enhanced dialogue functionality, but the driving factor of that tolerance might be the need to maintain epistemic flexibility in the face of signal-referent volatility. In the next section, we illustrate how interlocutors mutually develop shared conceptual spaces, epistemic systems 
that adapt at the same scale as the goals and needs of the interaction and that afford a certain tolerance for local ambiguity and misunderstanding.

\section{What do interlocutors construct?}

\section{Joint epistemic engineering}

An explanatory framework of human communication needs to clarify how inherently asymmetric individuals rapidly arrive at mutually understood mental constructs from ambiguous signals that are referentially contingent on the current communicative circumstances. Existing frameworks tend to minimize those fundamental communicative obstacles, e.g., signal ambiguity (Hasson et al., 2012; Rizzolatti \& Arbib, 1998), interpersonal asymmetry (Pickering \& Garrod, 2013), or type inadequacy (Gibson et al., 2019). We have recently argued, supported by neural evidence, that people overcome those obstacles because they solve, at each and every dialogue turn, a conceptual alignment challenge (Stolk et al., 2016). This alignment challenge entails that interlocutors continuously evaluate their conceptual structures for consistency with what is implied by the other in light of the ongoing interaction, like a reader needs to find and maintain consistent relationships between concepts conveyed in this contribution (e.g., links between Peirce, /bæyk/, and asymmetry). Here we zoom in on this epistemic engineering process, illustrating how interlocutors construct multilayered communicative signals to probe and give shape to their continually changing conceptual spaces. Using two conversational settings, one verbal and one non-verbal, we show that a signal can operate across different levels: to offer cues for aligning the space of possible interpretations across interlocutors (a Peirce-signal), while asking to be recognized as communicative (a Grice-signal) and reducing uncertainty over the identity of its referent (a Shannon-signal if the Peirce- and Grice-signals evoke the intended interpretational context). 
Furthermore, we show how those multi-layered signals are not processed in serial isolation. The contextual constraints suggested by the production of each Grice- and Peirce-signal accumulate and change as the interaction proceeds. By way of analogy, each signal production can be thought of as a stroboscopic flash, designed by the communicator to transiently illuminate her own rapidly shifting conceptual landscape for the benefit of the interlocutor. Across multiple turn-taking cycles, the interlocutors flash those strobes to offer a glimpse of each other's conceptual landscapes, so that they can be meshed together. The spectral qualities of the stroboscopic light can change over time, resolving finer details of the interlocutors' contextual framework as the interaction progresses successfully, or zooming back to a coarser granularity if a new dialogue is started. The integration window over multiple signals can also change as a function of interaction status, as when we open a dialogue confidently assuming mutual knowledge of an earlier conversation with the same interlocutor, or tentatively explore a newcomer with a pleasantry. Joint epistemic engineering is meant to emphasize that interlocutors are focused on controlling the shared conceptual landscape, rather than the consistency of signals or signal-referent mappings. By embedding signals in the shared conceptual space generated through the engineering process, interlocutors rapidly zoom in on relevant features of those signals, coordinate current reference points, and prepare the ground for anchoring new reference points. 


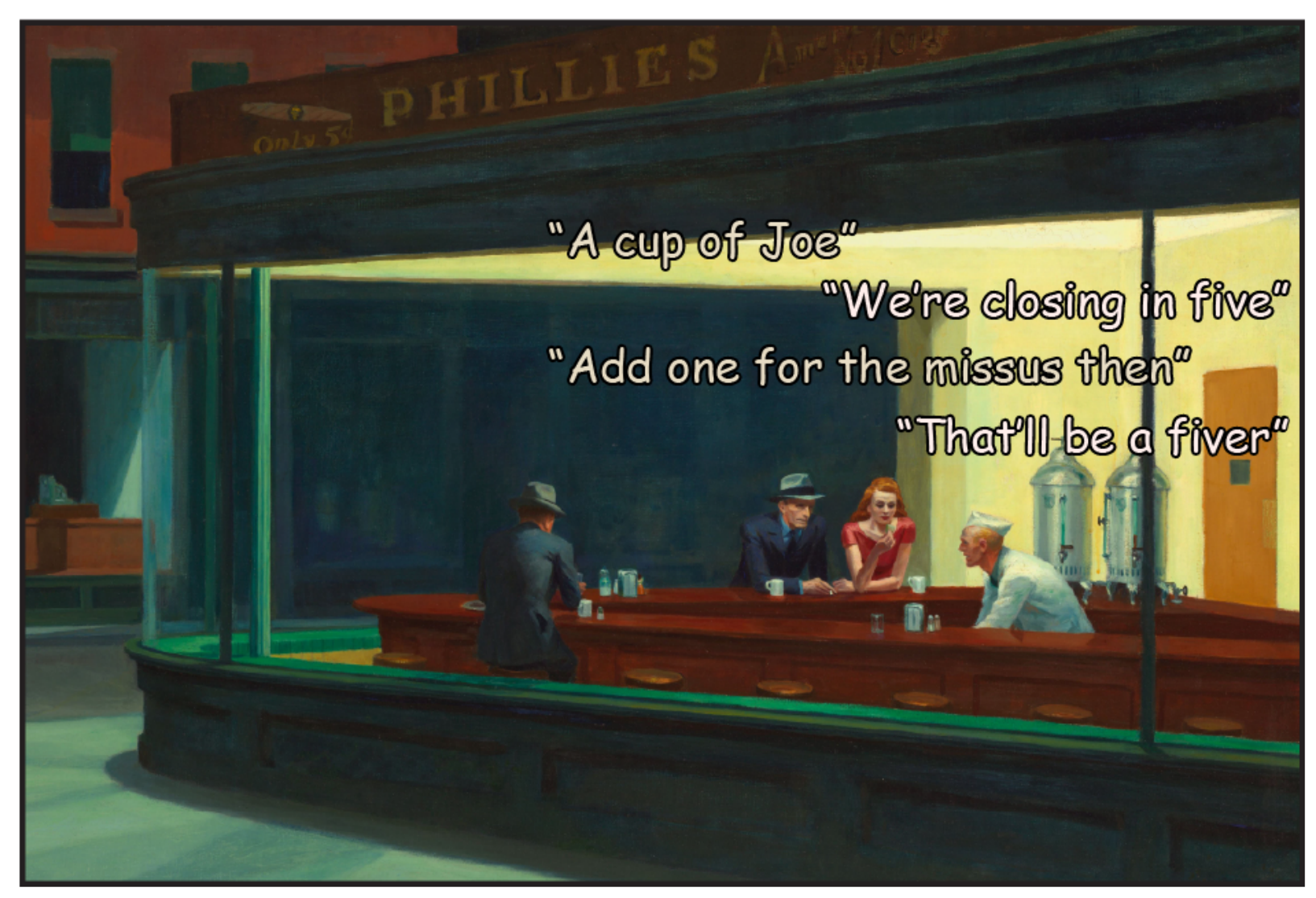

Figure 1. A prototypical human interaction, semantically incoherent but semiotically fluent. Artwork courtesy of the Art Institute of Chicago.

\section{Engineering a bar conversation}

It is easy to overlook how joint epistemic engineering underpins everyday interaction.

Consider the bar conversation shown in Figure 1. The flow and apparent straightforward understanding between customer and bartender might mask the interpretational constraints necessary to make sense of a dialogue laced with semantic incoherence and ambiguity. Even before voicing "A cup of Joe," the customer would need to build a scaffold of conceptual information that approximates the scaffold presumably used by the bartender to interpret his utterance (black graph structures in Figure 2A). The customer needs to select background knowledge that coffee can be ordered from bars; that bartenders may be familiar with slang words for coffee; and so on (blue background structure in Figure 2A). In fact, several 
possible-world scenarios might need to be prepared: the presumed bartender is a cleaner; the bar is already closed; etcetera. Accordingly, besides placing an order (a Shannon-signal), the customer's opening statement doubles as a tacit request to probe the conceptual scaffold shared with his interlocutor (a Peirce-signal), and to be recognized as such (a Grice-signal). The bartender's reply, "We're closing in five," despite bearing no semantic resemblance to the customer's opening utterance, suffices to address the customer's multi-layered request. He confirms his ability and willingness to process the order (addition of coffee to the conceptual space, Figure 2B) as well as to engage in the joint engineering process. Besides conveying recognition of communicative intent (a Grice-signal) and additional details about the bar (a Shannon-signal), the bartender's disclosure of the approaching closing time also operates as a tacit invitation to negotiate the customer's current request (e.g., "I'm not serving," or "I'm serving but drink fast") or make another (e.g., a drink for his female companion), i.e., a Peirce-signal. This multiplicity of functions illustrates how interlocutors use signals to simultaneously coordinate current and anchor new reference points in their conceptual frame, effectively coordinating a situated source of interpretational constraints for each other's intrinsically ambiguous signals. 

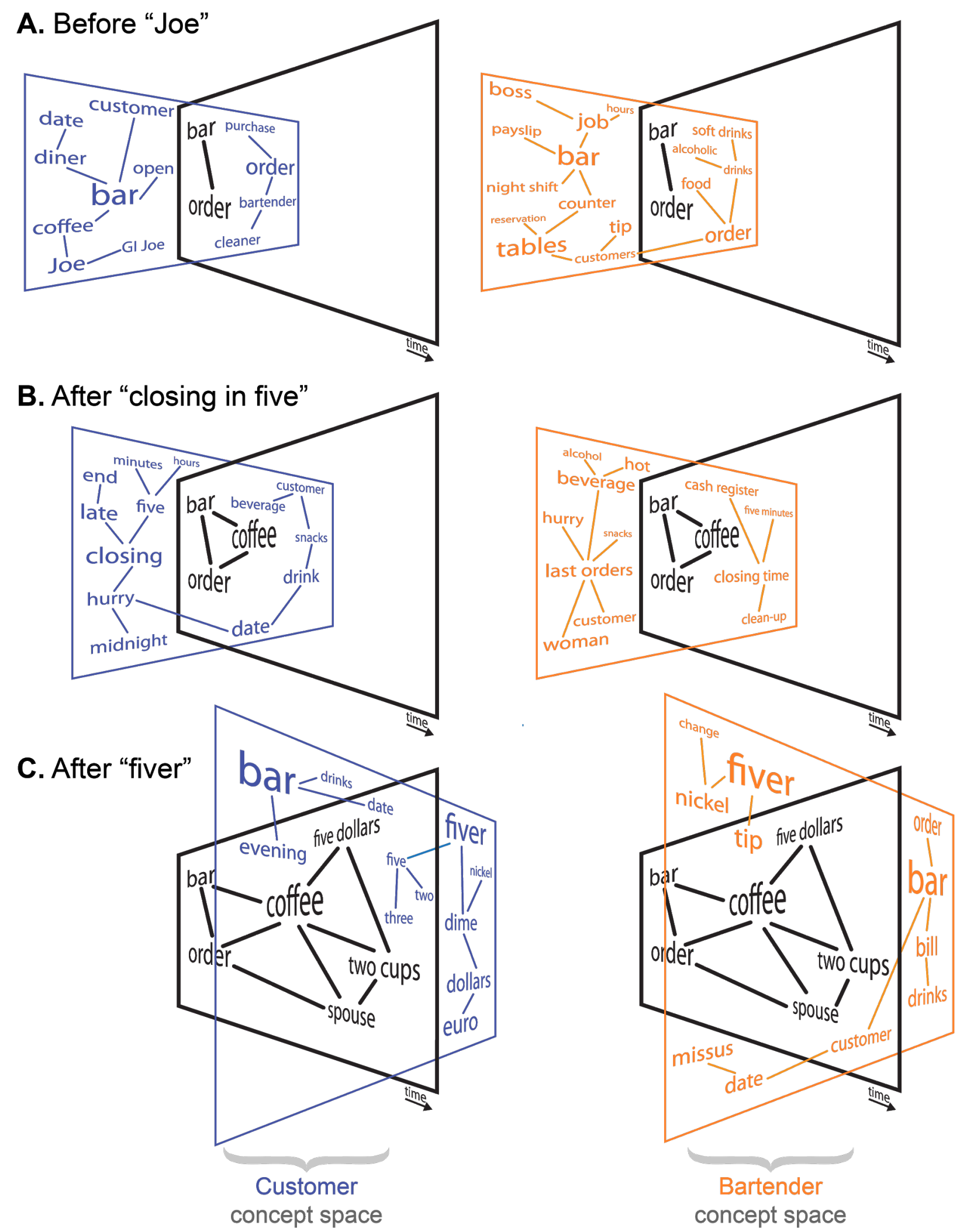

Figure 2. Epistemic dissections of three moments in the bar conversation shown in Figure 1, illustrating how dialogue requires people to continually build a scaffold of conceptual information that approximates the scaffold used by the other (forming a shared conceptual space, black graph structures). This joint engineering process kicks in even before the first signal is produced, and draws on individual background knowledge where possible (blue/orange graph structures). For instance, before voicing "A cup of Joe”, the customer needs to select 
background knowledge that coffee can be ordered from bars or that bartenders might be familiar with slang words for coffee, while also preparing alternative scenarios (blue background structure, A). Though semantically incoherent with the customer's opening statement, the bartender's reply, "We're closing in five", suffices to address the customer's request that he can and is willing to process the order (addition of coffee to the conceptual space, B). As interaction unfolds, the interlocutors construct their multi-layered signals to probe and give shape to their expanding conceptual spaces, keeping those spaces aligned with one another to provide a situated source of interpretational constraints (black structures, C).

This bar conversation also illustrates how joint epistemic engineering offers the possibility to fluidly reference pockets of readily shareable background knowledge (blue and orange graph structures in Figure 2), amalgamating potentially unrelated conceptual structures into a space of meanings and relationships between those structures. For instance, the customer's specification of "the missus" in his reply, "Add one for the missus then," offers the bartender with an unsolicited set of cues to the identity of his female companion, ground he could have expanded on in a subsequent exchange. Perhaps the innovation within the bartender's response, "That'll be a fiver," hints at an alignment on the level of nonchalance still, consistent with the customer's slang use. But on its own, the bartender's response references a separate pocket of background knowledge (concept of payment, orange structure in Figure 2C), knowledge he expects his interlocutor to also consider when hearing his response in the context of their current conceptual frame. Needless to say, that reference would have been out of place in other moments of the exchange (e.g., before the customer's order). Moreover, by exploiting the conceptual space they have jointly assembled and streamlined over their four utterances (black structures in Figure 2C), the interlocutors can rapidly generate plausible hypotheses about even novel signals, as when a customer would hear "fiver" for the first time. That epistemic inference would widely miss its target when working from the sensory data using context-free statistical regularities abstracted from speech/text corpora, as AIbased language processors do. Lacking a cognitive architecture for joint epistemic 
engineering, those artificial agents would fail to interpret "fiver" in the context of the customer's opening statement, instead limiting the search for relevant context to semantically, temporally, or arithmetically closer signals in the turn-taking sequence (e.g., "in five," "Add one").

\section{Engineering a communication system}

It could be argued that the previous example, while plausible, remains an armchair argument with no empirical evidence. In fact, even if the utterances had been recorded from a real diner, the conversational commentary would be purely speculative and rather descriptive. Fortunately, some researchers have managed to gather experimentally controlled observations on human communication, without losing sight of its core interactive features. The rationale of the approach is that when shared assumptions on what counts as a context and a signal are experimentally removed, then the production and interpretation of Grice and Peirce-signals will become more apparent, revealing the mechanisms of joint epistemic engineering in the process. Although the approach might seem to rely on experimentally contrived situations (see Figure 3 for an example, discussed in more detail below), similar challenges are common when communication cannot count on a shared idiom. Take the situation experienced by the Hawaiian people when visited by James Cook in 1779. As in many other "first encounters" between different groups (Leahy, 1936), people found ways to communicate reliably, and the British sailors spent a peaceful month on the island. Yet, shortly afterward, James Cook lost his life in a confrontation, apparently a consequence of miscommunications arising from a change of context, obvious to the Hawaiian people (the shift from the season of peace to the season of war) but completely opaque to the Western visitors. It is hardly surprising that during that episode, one of the crew members became sensitive to the fixed-code fallacy in Signs and Words (see epigraph). 
Several groups have recently started to re-create first encounters in the lab (without most of the risks) as a way to gain experimental access to core generative processes supporting human communicative abilities (de Ruiter et al., 2010; Galantucci, 2005; Scott-Phillips et al., 2009; Selten \& Warglien, 2007). This research program, under the general banner of “experimental semiotics” (Galantucci \& Garrod, 2011; Toni \& Stolk, 2019), has created various communication games where human participants interact using signals lacking a preexisting conventional use (e.g., artificial words, scribbly drawings). Importantly, while signal and context accessibility are experimentally controlled, great care is usually taken to preserve the minimal condition for studying human communication, i.e., the presence of two or more people in interaction. In our work, we have frequently used the "tacit communication game," in which two players converse on a 3-by-3 digital grid using the movements of a geometric shape (de Ruiter et al., 2010). In each round of the game, the two players have to jointly reproduce a target configuration of two shapes, one for each player (a circle, rectangle, or triangle). Crucially, however, the target configuration is shown to one of the players only, the sender, see Figure 3. Given that the other player, the receiver, cannot see this configuration, the sender needs to communicate the receiver's target position to the receiver while also ensuring that the final location and orientation of their own shape is as specified by the target configuration. The only means available to the sender for communicating with the receiver is by moving the sender's own shape around the grid using horizontal and vertical translations, and $90^{\circ}$ clockwise rotations controlled by button presses on a handheld game controller. The only means available to the receiver for completing the configuration is by inferring the target location and orientation of her own shape based on the movements of the sender and positioning it accordingly using a second handheld game controller. As the receiver cannot ask for clarification, the only indication of whether her interpretation of the sender's signals 
was correct is feedback on the token's position received at the end of each trial. The sender not only has to monitor whether the receiver got the target location, but also whether she is adhering to the tacitly agreed-upon communicative strategy or making any changes in the way communicative signals have been used so far.

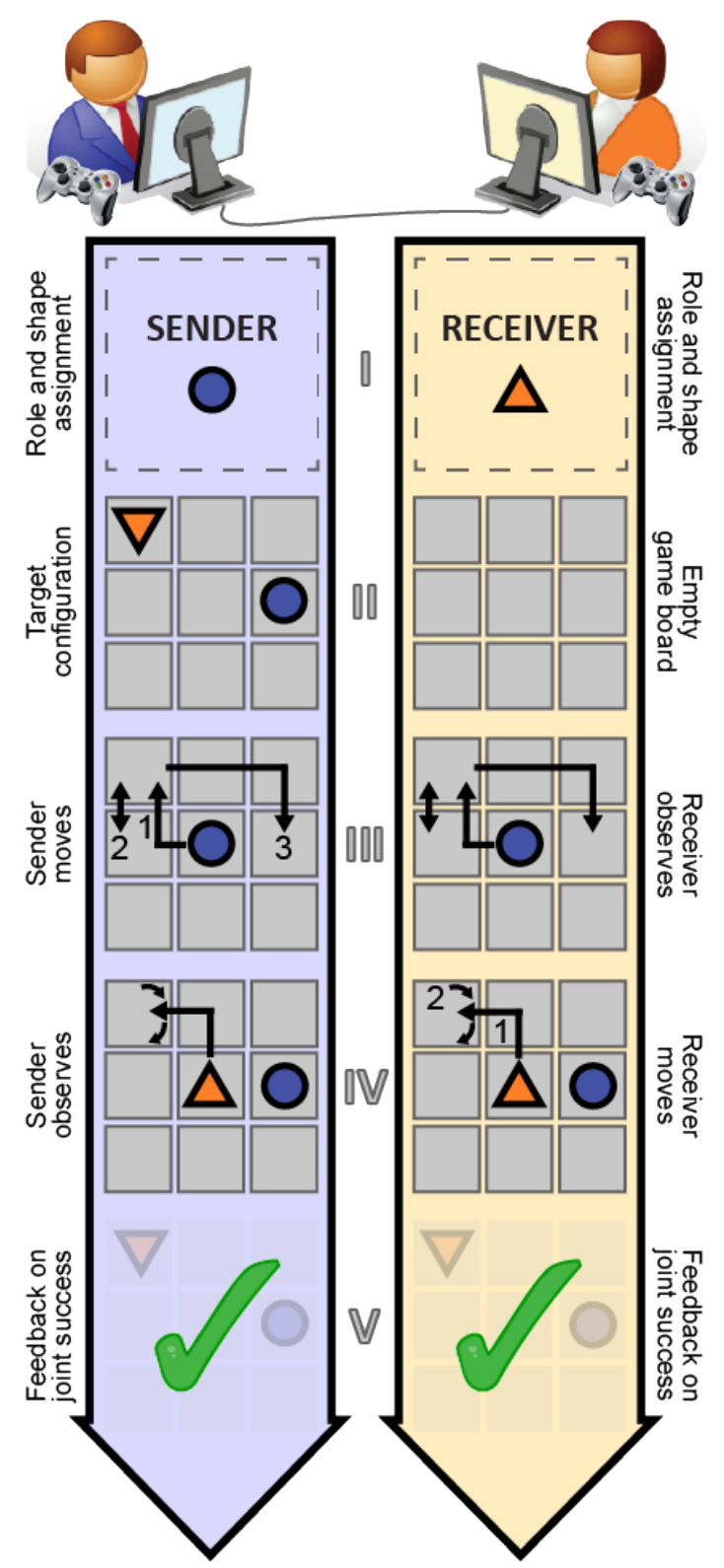

Figure 3. Tacit communication game. The joint goal of sender (left) and receiver (right) is to reproduce a target configuration of their two given shapes on a digital game board. This target configuration is shown to the sender only (event II). Given that the receiver cannot see the target configuration, a successful interaction requires the sender and receiver to construct and comprehend how the behavior of the sender's shape indicates the location and orientation of the receiver's shape (event III). Feedback is presented to both players with a green checkmark 
or red cross indicating joint success or failure (event V). A benefit of the novel communicative setting is that the shape behaviors lack conventional meaning, providing reliable access to the core process of how two humans converge on a shared meaning for a signal. An online version of the game can be found at www.MutualUnderstanding.nl.

There are no a priori correct communication strategies in the tacit communication game, nor can the receiver solve the task by simply reproducing the movements of the sender's shape. Analogous to everyday multimodal communication involving ambiguous signals, the receiver needs to rapidly zoom in on relevant features of the sender's movements and infer a possible referent (a shape configuration) on the basis of those features. Example features include brief movement "pauses" on the receiver's target location, distinguishing it from other locations visited on the grid, as well as rotational movements, directional movements, and repeated stepping in and out from the receiver's target location ("wiggling"), with the latter three movement types typically used to indicate target orientation. A type-centered framework might assign those movement features to signals' referents as if they constitute optimal solutions to (parts of) a problem, but this would miss the local principles that govern their interpretations (Garrod \& Anderson, 1987). For instance, it cannot be determined from a wiggling behavior alone whether it is intended to emphatically indicate the direction in which the receiver's shape needs to point, or whether the number of wiggles corresponds to the number of clockwise rotations the receiver needs to make to reach the target orientation, with some pairs using both strategies during a single game. These observations indicate how in this game, as in the bar conversation, the trajectory from a signal to its referent is contingent on the communicative context people develop together.

To illustrate how joint epistemic engineering could support rapidly understood new signalreferent trajectories, even when a different trajectory exists for a signal, we here turn to the 
case of the "missing wiggle." Before doing so, it should be mentioned that to drive interlocutors to continuously develop and coordinate their shared conceptual spaces, the tacit communication game increases the task difficulty across successive interactions. This is achieved by introducing deliberate mismatches between the geometric characteristics of the pairs' shapes, including situations where a receiver's target orientation is incompatible with the pair's current strategy. For instance, if a sender was able to successfully communicate the target orientation of the receiver's shape by rotating their own shape, this strategy would be negated when the sender's shape changed to a circle and the receiver's to a triangle. The sender would then have to find a new way to indicate the target orientation of the receiver's shape because rotations of a circle are not visible. A further level of difficulty is introduced by maintaining the same circle-triangle shape combination but having the receiver's triangle point outside the grid. If the sender had previously used a signal that involved repeatedly stepping in and out of the receiver's target location in the direction of the target orientation ("wiggling," number 2 double-headed arrow in Figure 3), an interaction where the receiver's triangle pointed outside of the grid would be impossible to solve using that signal.

Returning to our case, several pairs started to use a "pause" strategy to indicate that the triangle needed to point outside of the grid. Those pairs had previously converged on using movement pauses on receiver target locations to selectively indicate the communicative relevance of those grid locations, thus with no obvious role for pauses in conveying target orientation other than the default orientation. Hence, through a lens of associative learning, the use of a pause strategy would unambiguously mean that a triangle needed to be positioned in its default upward position, rather than outward-facing. How then could pairs have rapidly converged on a new use for an existing signal in the absence of any failures? A closer look at a pair's recent communicative history hints at a conceptual shift as to what movement 
features the pair mutually considered relevant in their communication. Specifically, the pair had recently converged on using a wiggle strategy to indicate the direction in which a triangle needed to point (as in Figure 3), applying this strategy even in cases where a triangle needed to point upward and previously a pause would have sufficed. In doing so, wiggling had not only become the prevalent feature for indicating target orientation in this pair, but the conceptual shift in feature relevance had implicitly relaxed the relationship between pauses and default orientations. Consequently, pauses could be introduced by the sender to signify orientation in situations where he could safely assume that the receiver would infer wiggles could not (i.e., a missing wiggle, see dashed lines in Figure 4), such as with outward-facing triangles. This epistemic inference illustrates how a shift in conceptual space can cause a signal to acquire a different meaning in (apparently) a single instance, as when a pause becomes a missing wiggle. 

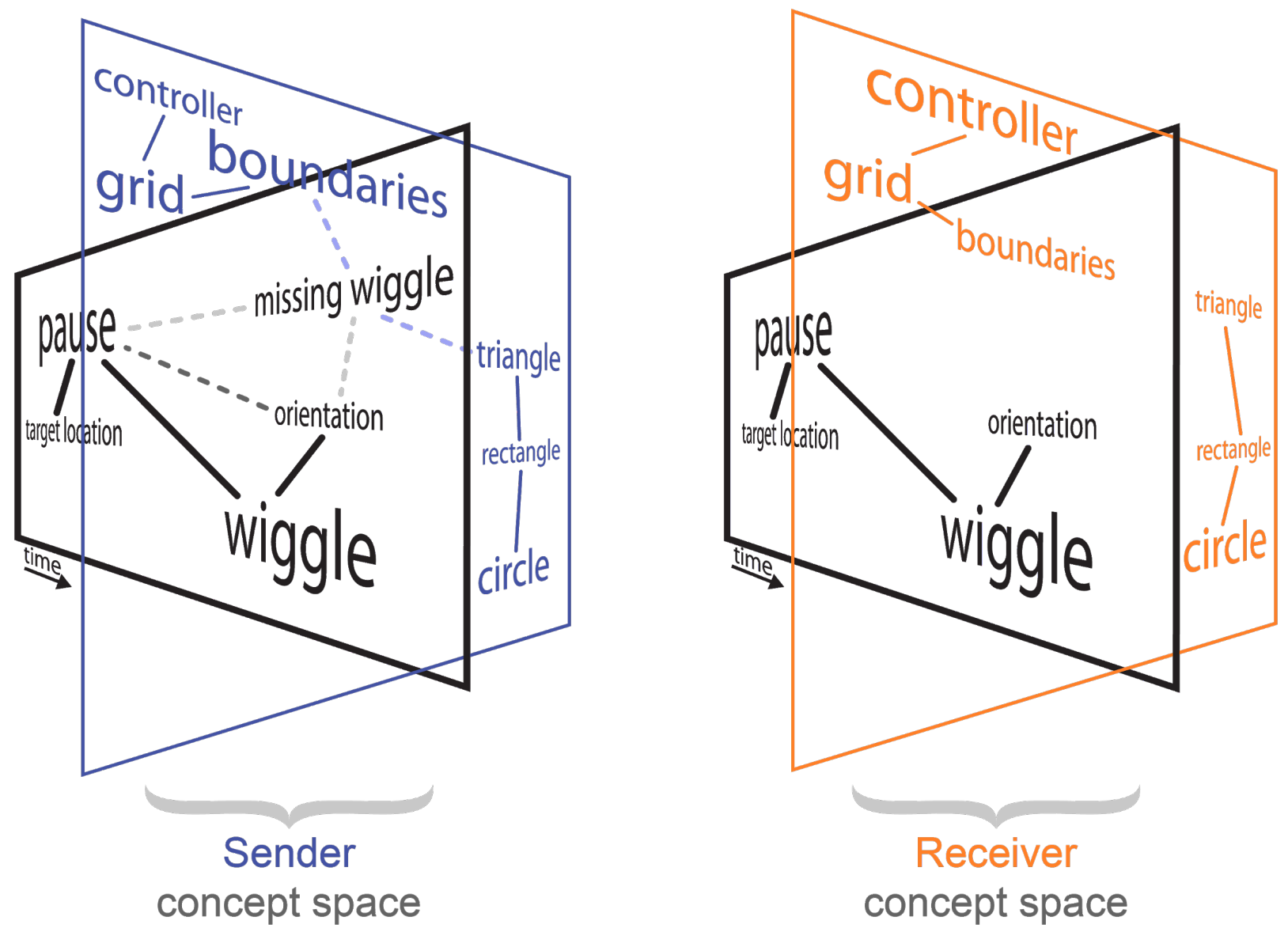

Figure 4. Epistemic dissection capturing the moment a signal acquires a new meaning in the tacit communication game. The figure on the left captures the moment the sender used a pause, previously used exclusively to signal target location (solid black connection), to additionally signal orientation (dashed grey connection), a function previously reserved for wiggles (solid black connection). To understand this new use for behaviorally identical pauses (pause as a "missing wiggle"), the receiver would need to infer the implied change in their conceptual space driving that proposal. For instance, he would need to infer that the sender faced a situation in which wiggles could no longer be used to signal orientation, such as with an outward-facing triangle at the grid's boundary. He would also need to consider conceptual shifts supporting that new wiggle-less orientation-signaling strategy (dashed connections radiating out from "missing wiggle", left figure). If he then manages to convey agreement about that epistemic inference to the sender, the players can strengthen or add connections in their conceptual space supporting the sender's proposal (dashed grey connections on the left becoming solid in both left and right figures).

\section{What does the brain care about?}

The previous sections provide prima facie evidence for moving beyond views of human communication as a signal coding-decoding problem. Whether during ordinary conversations 
or during "first encounters" deprived of a common language (Figures 2 and 4), interlocutors need to construct their signals to probe and give shape to their conceptual frame of reference, keeping it aligned with the current situation and with one another. By embedding signals in the shared conceptual space generated through this engineering process, interlocutors rapidly resolve the multiple ambiguities in every signal. Here we discuss recent work that has begun to empirically interrogate these theoretical issues at the implementation level by combining neuroscientific techniques with controlled studies of live social interaction, i.e., the tacit communication game outlined above. To date, there are three major insights.

First, both production and comprehension of communicative signals are supported by a rightlateralized frontotemporal network, shown in Figure 5A (Stolk et al., 2013). This network has been shown to contribute to pragmatic and mental state inferences when communicating with linguistic means, suggesting a role for right frontotemporal cortex in embedding utterances in their conversational context (Bašnáková et al., 2014; Beeman, 1993; Sabbagh, 1999). Second, neural activity in this right-lateralized network predates in time the production and comprehension of communicative signals, i.e., before the initial dashed lines in Figure 5A. This observation fits with the notion that communicative behaviors are selected and interpreted within the conceptual frame of reference people in dialogue develop together. Interference studies of anterior frontal and posterior temporal nodes of the network confirm and qualify the contribution of those cortical regions. Ventromedial prefrontal lesion patients, known for their social conduct deficits (Beer et al., 2006), were found to lack the specific ability to constrain their ongoing communication with knowledge implied by the current communicative circumstances, including the presumed abilities of an interlocutor (Stolk et al., 2015). Transient disruption of neural function in the right posterior superior temporal sulcus diminishes the ability to constrain the interpretation of communicative behaviors using 
knowledge abstracted from the recent communicative history (Stolk, Noordzij, Volman, et al., 2014). These causal observations emphasize the constraining role of contingently updated conceptual knowledge in social interaction.

Third, activity in one subregion of the right-lateralized frontotemporal network, the superior temporal gyrus (STG), becomes synchronized across interlocutors due to the accumulation of shared conceptual knowledge (Stolk, Noordzij, Verhagen, et al., 2014). This neural synchronization occurred only when interlocutors developed shared communicative solutions, and at a temporal scale closer to communicative episodes than the behaviors themselves, see Figure 5B. Conversely, neural synchronization was reduced over communicative episodes that did not require interlocutors to coordinate shared knowledge. These observations define a neural basis for the notion that interlocutors mutually coordinate their conceptual knowledge updates during social interaction. 
A.

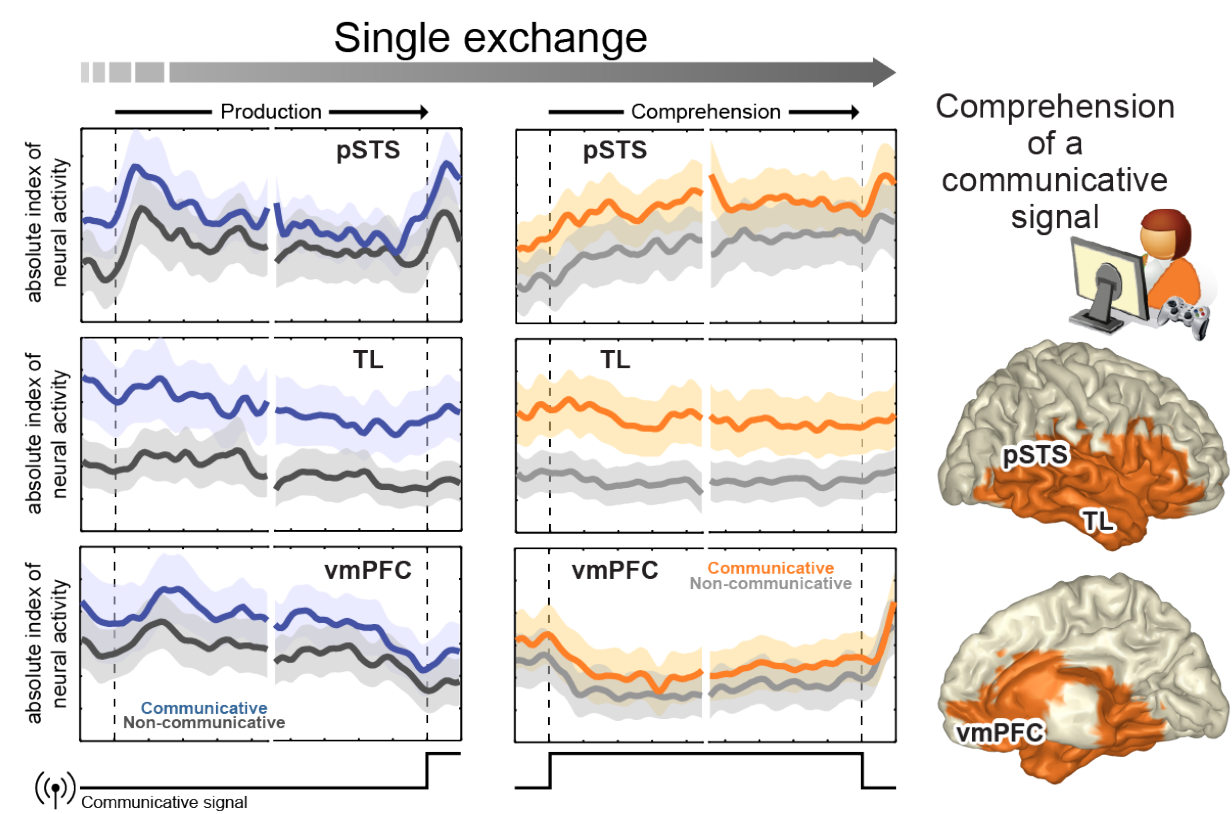

B.

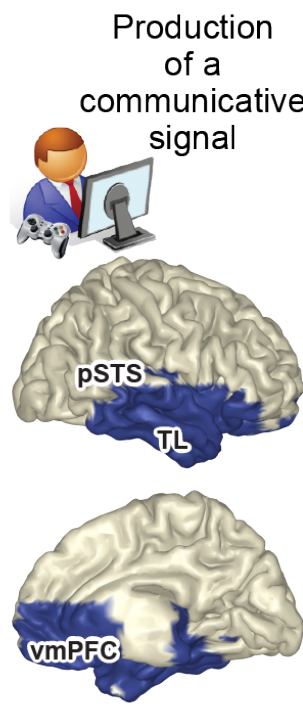

communicative

inal

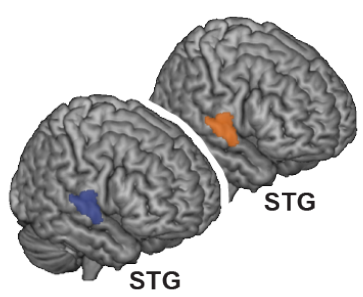

Multiple exchanges

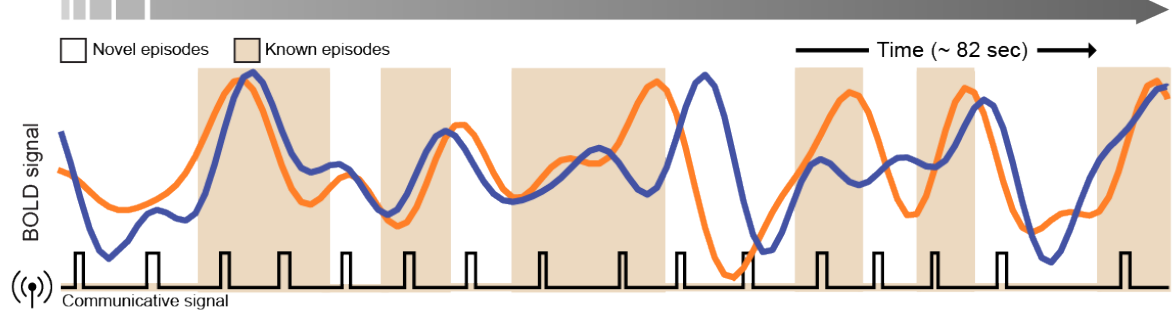

Figure 5. Neural dynamics of sharing conceptual spaces within a single communicative exchange (A) and over multiple exchanges (B). Brain regions of a right-lateralized frontotemporal network supporting both production and comprehension of communicative signals (blue and orange, respectively) are already upregulated before a communicative signal is produced or comprehended (before the initial dashed in lines in A). Activity in one subregion, the superior temporal gyrus (STG, shown in B), tracked communicative success throughout the experiment (not shown) and was coherent across interlocutors at a temporal scale independent from individual communicative signals (blue and orange traces). Adapted from (Stolk et al., 2016).

The picture that emerges from these brain observations is one in which the meaning of a communicative signal is not a property of the signal, nor of individuals interpreting that signal. Rather, meaning is a property of conceptual knowledge inferred from prior beliefs and interactive behaviors embedded in the recent communicative history and kept aligned with the unfolding interaction to constrain communication. What neurophysiological mechanism 
allows a sustained yet adjustable influence of conceptual knowledge on transient signal production and comprehension? A clue is provided by the right-hemispheric frontotemporal structures found to support communication. Densely spaced neuronal populations with overlapping dendritic fields and thin axonal myelination endow those frontotemporal association areas with a relatively large-world architecture compared to their left hemisphere homologs (Hutsler \& Galuske, 2003; Jung-Beeman, 2005). Highly interconnected populations of neurons might confer conceptual processing at varying levels of abstraction, offering a neuroanatomical infrastructure to flexibly scaffold and search for relevant context in communication.

Another clue is provided by the spectrotemporal nature of neural activity observed over the frontotemporal cortex. Temporally, frontotemporal regions are tonically upregulated during communicative interactions, showing strikingly similar phasic neural dynamics during the production and comprehension of both communicative and non-communicative events (blue/orange vs. grey traces in Figure 5A). This observation is consistent with an increasing body of evidence showing that contextual demands can modulate ongoing neural activity yet retain responsiveness to event-related neural processing (Abitbol et al., 2015). Indeed, tonic upregulation had measurable behavioral consequences on communicative performance (Stolk et al., 2013). Spectrally, the neural upregulation over the frontotemporal cortex had an extremely broad profile (not shown in Figure 5). Broadband shifts of neural activity are thought to reflect changes in the mean firing rates of neuronal populations, and their antecedence to observable events might be instrumental to integrate driving afferences with contextual information. Specifically, ongoing contextual inputs can temporarily hold selective neurons in an excitable state, increasing the probability of those neurons propagating event-related inputs, effectively integrating information associated with the input 
streams (Smith et al., 2013). This neuronal mechanism, based on upregulated broadband neural activity in the right frontotemporal cortex, might provide a neural marker of the conceptual frame of reference people build together in communication (Stolk et al., 2013).

\section{What could possibly go wrong?}

The theoretical and empirical observations presented in this chapter might be seen as exceptional situations that do not generalize to the daily interactions of a linguistic community. On the contrary, joint epistemic engineering might be relevant to understand communicative alterations despite a relatively intact command of language. For instance, individuals with damage to the right hemisphere may respond appropriately to clearly structured questions requiring specific answers, e.g., "Where do you live?" Yet they produce irrelevant and verbose answers (in a Gricean sense) to open-ended questions such as "What did you do yesterday?" Patients seem unable to assemble their utterances into a coherent narrative, instead wending their way through a maze of disassociated detail, without answering the question (Myers, 1979). Comparable deficits have been noted in patients suffering frontotemporal dementia, where coherent conversations, as well as intrinsically motivated social relationships, are affected when the frontal lobe and right temporal pole degenerate (Mates et al., 2013). These clinical observations converge with the brain observations detailed above in linking a right-lateralized frontotemporal network to the processing of conceptual knowledge of the communicative context.

Ultimately, it will be important to make systematic pathophysiological comparisons between patient groups with communicative alterations, potentially isolating cognitive phenotypes that unify apparently disparate disorders, e.g., schizophrenia, autism spectrum disorder, frontotemporal dementia (Ibáñez et al., 2018). For instance, it might emerge that patients with 
either of those disorders have problems in creating and maintaining a shared conceptual space with an interlocutor. Recent evidence from our group suggests that this alteration is present in cognitively able adults on the autism spectrum (Wadge et al., 2019). Despite otherwise indistinguishable performance from neurotypical pairs, autistic pairs had lower communicative success in the tacit communication game. This communicative impairment was not simply a consequence of reduced cognitive flexibility or social motivation, as autistic individuals showed a similar propensity to change their signals following a misunderstanding with their partner. However, they struggled to assign meanings to signals based on the unique communicative context established through past interaction with their partner, showing more individual exploration when constructing those signals. As seen in the largely segregated interaction spaces between autistic individuals in Figure 6 (blue vs. orange clusters), neurotypical pairs navigate open-ended communicative situations by continuously considering and aligning to the frame of reference defined by signals embedded in their communicative history (in a Peircean sense). These observations explain, for instance, why autistic individuals experience difficulties in everyday context-dependent situations such as ironic or sarcastic conversation, but succeed in tests where they can capitalize on stereotyped cues to resolve linguistic ambiguities (Hahn et al., 2015). 


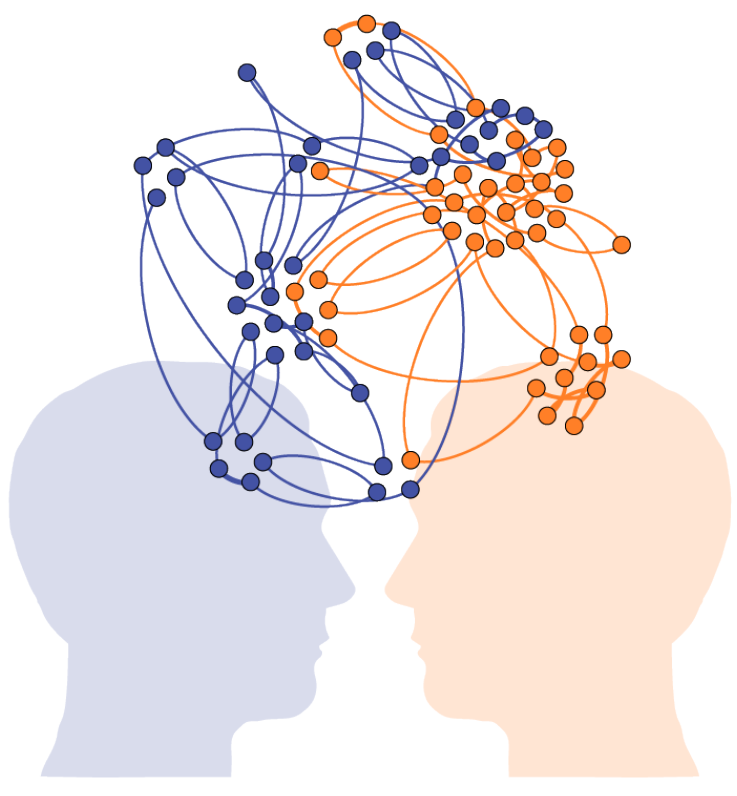

ASD interaction space

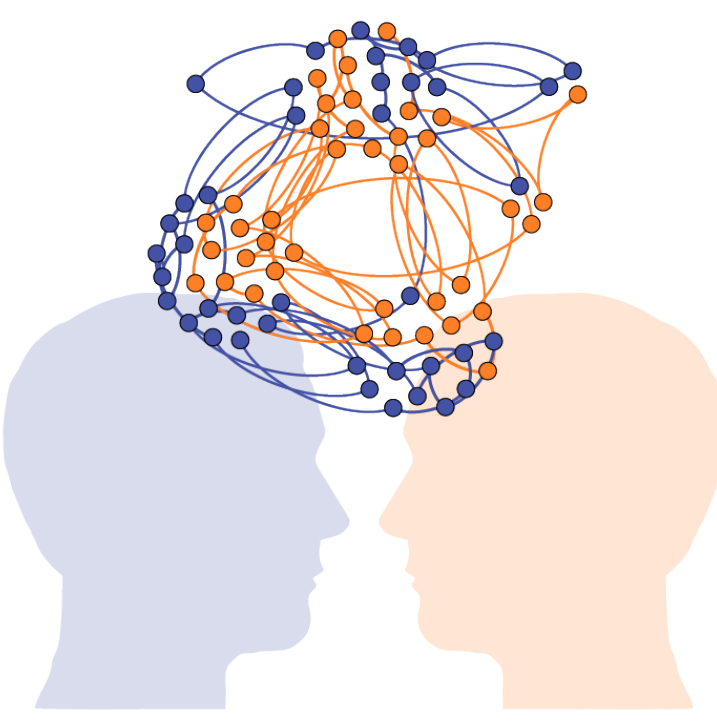

Neurotypical interaction space

Figure 6. Network visualizations of pairwise interaction trajectories by an autistic (left) and a neurotypical pair (right). The nodes represent communicative signals constructed by two individuals during a series of interactions in the tacit communication game. The nodes are clustered to show signals that were used repeatedly. The colored edges connect each individual's consecutively produced signals throughout the interaction (blue for one individual, orange for the other). It can be seen that autistic individuals showed more individual exploration when constructing signals, as indicated by relatively large clusters of individually used signals and small clusters of jointly used signals. By contrast, the neurotypical pair navigated the interaction space by continuously considering and aligning to recent signals from their partner, embedding those signals in a strongly interconnected space of meaning and relationships between one another's signals. ASD, Autism Spectrum Disorder. Adapted from (Wadge et al., 2019).

Investigating the role of communicative context in language development is another research direction that, in our opinion, will provide important insights into the interplay between stereotyped and fleeting conceptual structures in epistemic engineering. For instance, while language processing is largely left-lateralized throughout life, necessary contributions from the right hemisphere may be seen early in life, gradually decreasing through childhood. Moreover, the right hemisphere may support basic language skills when the left hemisphere is compromised (Olulade et al., 2020). To date, there is no evidence for the opposite, with the 
left hemisphere seemingly not able to compensate for lost conversational abilities after early right hemisphere damage. As noted elsewhere (Sabbagh, 1999), this pattern of recovery is reminiscent of the pattern of linguistic and communicative development in autism. Vocabulary development is delayed but occurring in autistic children, yet communicative deficits persist through adulthood, as they would in right hemisphere patients. We infer from its comparably small-world architecture that the left hemisphere is less able to cope with the flexibility demands of everyday interaction. Widely spaced neuron populations with little overlapping dendritic fields and greater levels of myelination endow the left hemisphere with functional stability and efficiency in terms of perturbation-resistant network hubs and shorter path lengths. Accordingly, the left hemisphere might be better equipped to generalize recurrent patterns of association, when scaffolded by the conceptually flexible right hemisphere. These stereotyped dependencies, in turn, help communication by providing access to semantic structures and cues to a meaning (blue and orange background structures of Figure 2).

\section{Conclusion}

We have introduced joint epistemic engineering, a neurosemiotic account of how inherently asymmetric interlocutors can communicate effectively despite using signals that are referentially contingent on the current communicative circumstances. The basic insight is that interlocutors use multi-layered communicative signals as a tool to organize and align their conceptual structures during social interaction. Besides reducing uncertainty over the identity of a referent in the interlocutors' minds (a Shannon-signal), a multi-layered signal asks interlocutors to manage that declaration of communicative intent (a Grice-signal) and to coordinate the space of possible interpretations across interlocutors (a Peirce-signal). Accordingly, joint epistemic engineering offers the possibility to establish a communicative 
context in the presence of highly ambiguous signals, as well as to fluidly contextualize background knowledge relevant to the current situation, amalgamating potentially unrelated conceptual structures into a space of meanings and relationships between those structures. The hypothesized dependencies between those semiotic relations open the way to precisely characterize neural mechanisms that can implement the construal of meaning during interactive human communication. Future work should aim to understand how the elusive conceptual space generated during communication is instantiated in our brains, how its neural implementation is altered in disorders of human communication, and how joint epistemic engineering can bootstrap communication when infants learn to use linguistic signals in their daily interactions.

\section{References}

Abitbol, R., Lebreton, M., Hollard, G., Richmond, B. J., Bouret, S., \& Pessiglione, M. (2015). Neural mechanisms underlying contextual dependency of subjective values: Converging evidence from monkeys and humans. The Journal of Neuroscience: The Official Journal of the Society for Neuroscience, 35(5), 2308-2320.

Bašnáková, J., Weber, K., Petersson, K. M., van Berkum, J., \& Hagoort, P. (2014). Beyond the language given: The neural correlates of inferring speaker meaning. Cerebral Cortex, 24(10), 2572-2578. doi: 10.1093/cercor/bht112

Beeman, M. (1993). Semantic processing in the right hemisphere may contribute to drawing inferences from discourse. Brain and Language, 44(1), 80-120.

Beer, J. S., John, O. P., Scabini, D., \& Knight, R. T. (2006). Orbitofrontal cortex and social behavior: Integrating self-monitoring and emotion-cognition interactions. Journal of Cognitive Neuroscience, 18(6), 871-879.

Brennan, S. E., Galati, A., \& Kuhlen, A. K. (2010). Two minds, one dialog: Coordinating 
speaking and understanding. In B. H. Ross (Ed.), The psychology of learning and motivation: Advances in research and theory (pp. 301-344). Cambridge: Elsevier Academic Press. doi: 10.1016/S0079-7421(10)53008-1

Carpendale, J. I. M., \& Lewis, C. (2004). Constructing an understanding of mind: The development of children's social understanding within social interaction. The Behavioral and Brain Sciences, 27(1), 79-96; discussion 96-151.

Clark, H. H. (1996). Using language. Cambridge: Cambridge University Press. doi: $10.1017 /$ cbo9780511620539

Clark, H. H. (1997). Dogmas of understanding. Discourse Processes, 23(3), 567-598. doi: $10.1080 / 01638539709545003$

Clark, H., \& Marshall, C. (1981). Elements of discourse understanding. In Aravind K. Joshi, Bonnie L. Webber, \& Ivan A. Sag (Eds.) Definite knowledge and mutual knowledge, (pp. 10-63). Cambridge: Cambridge University Press.

Conway, B. R. (2012). Color consilience: Color through the lens of art practice, history, philosophy, and neuroscience. Annals of the New York Academy of Sciences, 1251, 7794.

Danchin, E., Giraldeau, L. A., Valone, T. J., \& Wagner, R. H. (2004). Public information: From nosy neighbors to cultural evolution. Science, 305(5683), 487-491. doi: $10.1126 /$ science. 1098254

Dawkins, R., \& Krebs, J. R. (1978). Animal signals: Information or manipulation. In J. R. Krebs \& N. B., \& Davies (Eds.), Behavioural ecology: An evolutionary approach, (pp. 282-309). Oxford: Blackwell Scientific.

Deacon, T. W. (2018). Beneath symbols: Convention as a semiotic phenomenon. In S. C. Hayes \& D. S. Wilson (Eds.), Evolution \& contextual behavioral science: A reunification. (pp. 1-15). California: New Harbinger Publications. 
de Ruiter, J. P., Noordzij, M. L., Newman-Norlund, S., Newman-Norlund, R., Hagoort, P., Levinson, S. C., \& Toni, I. (2010). Exploring the cognitive infrastructure of communication. Interaction Studies, 51-77. doi: 10.1075/bct.45.04rui

Dingemanse, M., Roberts, S. G., Baranova, J., Blythe, J., Drew, P., Floyd, S., Gisladottir, R. S., Kendrick, K. H., Levinson, S. C., Manrique, E., Rossi, G., \& Enfield, N. J. (2015). Universal principles in the repair of communication problems. PloS One, 10(9), e0136100. doi: 10.1371/journal.pone.0136100

Elman, J. L. (2004). An alternative view of the mental lexicon. Trends in Cognitive Sciences, 8(7), 301-306.

Galantucci, B. (2005). An experimental study of the emergence of human communication systems. Cognitive Science, 29(5), 737-767.

Galantucci, B., \& Garrod, S. (2011). Experimental semiotics: A review. Frontiers in Human Neuroscience, 5. doi: 10.3389/fnhum.2011.00011

Galantucci, B., Langstein, B., Spivack, E., \& Paley, N. (2020). Repair Avoidance: When faithful informational exchanges don't matter that much. Cognitive Science, 44(10), e12882. doi: $10.1111 / \operatorname{cogs} .12882$

García, A. M., Franco-O’Byrne, D., \& Ibáñez, A. (2020). Neurosemiotics: Blurbing a field beyond the “two cultures divide.” In L'. Lacková, C. J. Rodríguez H., \& K. Kull (Eds.), Gatherings in biosemiotics XX, (pp. 92-97). Tartu: University of Tartu press.

Garrod, S., \& Anderson, A. (1987). Saying what you mean in dialogue: A study in conceptual and semantic co-ordination. Cognition, 27(2), 181-218.

Gaskell, G. M. (2007). The Oxford Handbook of Psycholinguistics. Oxford: Oxford University Press.

Gibson, E., Futrell, R., Piantadosi, S. T., Dautriche, I., Mahowald, K., Bergen, L., \& Levy, R. (2019). How efficiency shapes human language. Trends in Cognitive Sciences, 23(12), 
1087.

Grice, H. P. (1957). Meaning. The Philosophical Review, 66(3), 377-388. doi: $10.2307 / 2182440$

Hahn, N., Snedeker, J., \& Rabagliati, H. (2015). Rapid linguistic ambiguity resolution in young children with autism spectrum disorder: Eye tracking evidence for the limits of weak central coherence. Autism Research: Official Journal of the International Society for Autism Research, 8(6), 717-726.

Harris, R. (1998). Introduction to Integrational Linguistics. Oxford: Pergamon Press.

Hasson, U., Ghazanfar, A. A., Galantucci, B., Garrod, S., \& Keysers, C. (2012). Brain-tobrain coupling: a mechanism for creating and sharing a social world. Trends in Cognitive Sciences, 16(2), 114-121.

Hutsler, J., \& Galuske, R. A. W. (2003). Hemispheric asymmetries in cerebral cortical networks. Trends in Neurosciences, 26(8), 429-435.

Ibáñez, A., García, A. M., Esteves, S., Yoris, A., Muñoz, E., Reynaldo, L., Pietto, M. L., Adolfi, F., \& Manes, F. (2018). Social neuroscience: undoing the schism between neurology and psychiatry. Social Neuroscience, 13(1), 1-39.

Jefferson, G. (1988). On the sequential organization of troubles-talk in ordinary conversation. Social Problems, 35(4), 418-441. doi: 10.2307/800595

Jung-Beeman, M. (2005). Bilateral brain processes for comprehending natural language.

Trends in Cognitive Sciences, 9(11), 512-518. doi: 10.1016/j.tics.2005.09.009

Kirby, S., Griffiths, T., \& Smith, K. (2014). Iterated learning and the evolution of language. Current Opinion in Neurobiology, 28, 108-114.

Kull, K. (2020). Codes: Necessary, but not sufficient for meaning-making. Constructivist Foundations. Constructivist Foundations, 15(2), 137-139.

Leahy, M. (1936). The Central Highlands of New Guinea. The Geographical Journal, 87(3), 
229. doi: $10.2307 / 1786763$

Levinson, S. (2006). On the human interactional engine. In N. Enfield \& S. Levinson (Eds.), Roots of human sociality. (pp. 39-69). Oxford: Berg.

Lewis, D. K. (1969). Convention: A Philosophical Study. Princeton: Blackwell Publishers. Mates, A. W., Mikesell, L., \& Smith, M. S. (2013). Language, Interaction, and Frontotemporal Dementia: Reverse Engineering the Social Mind. Sheffield: Equinox. Mayr, E. (1999). Systematics and the Origin of Species, from the Viewpoint of a Zoologist. Cambridge, MA: Harvard University Press.

Misyak, J. B., Melkonyan, T., Zeitoun, H., \& Chater, N. (2014). Unwritten rules: Virtual bargaining underpins social interaction, culture, and society. Trends in Cognitive Sciences, 18(10), 512-519.

Myers, P. S. (1979). Profiles of communication deficits in patients with right cerebral hemisphere damage: Implications for diagnosis and treatment. Clinical Aphasiology: Proceedings of the Conference 1979, 38-46.

Noveck, I. A., \& Reboul, A. (2008). Experimental pragmatics: A Gricean turn in the study of language. Trends in Cognitive Sciences, 12(11), 425-431.

Olulade, O. A., Seydell-Greenwald, A., Chambers, C. E., Turkeltaub, P. E., Dromerick, A. W., Berl, M. M., Gaillard, W. D., \& Newport, E. L. (2020). The neural basis of language development: Changes in lateralization over age. Proceedings of the National Academy of Sciences, 201905590. doi: 10.1073/pnas.1905590117

Peirce, C. S. (1931). In Hartshorne, Charles Weiss, Paul Burks, Arthur W. (Eds.), Collected papers of Charles Sanders Peirce. New York: Thoemmes Continuum.

Pickering, M. J., \& Garrod, S. (2013). An integrated theory of language production and comprehension. The Behavioral and Brain Sciences, 36(4), 329-347.

Port, R. F. (2010). Language as a social institution: Why phonemes and words do not live in 
the brain. Ecological Psychology, 22(4), 304-326. doi: 10.1080/10407413.2010.517122

Rendall, D., \& Owren, M. J. (2013). Communication without meaning or information:

Abandoning language-based and informational constructs in animal communication theory. In U. E. Stegmann (Ed.), Animal communication theory, (pp. 151-188).

Cambridge: Cambridge University Press.

Rizzolatti, G., \& Arbib, M. A. (1998). Language within our grasp. Trends in Neurosciences, 21(5), 188-194.

Sabbagh, M. A. (1999). Communicative intentions and language: Evidence from righthemisphere damage and autism. Brain and Language, 70(1), 29-69.

Sacks, H., Schegloff, E. A., \& Jefferson, G. (1974). A simplest systematics for the organization of turn-taking for conversation. Language, 50(4), 696. doi: 10.2307/412243

Schelling, T. C. (1960). The Strategy of Conflict. Cambridge, MA: Harvard University Press.

Scott-Phillips, T. C., Kirby, S., \& Ritchie, G. R. S. (2009). Signalling signalhood and the emergence of communication. Cognition, 113(2), 226-233.

Selten, R., \& Warglien, M. (2007). The emergence of simple languages in an experimental coordination game. Proceedings of the National Academy of Sciences of the United States of America, 104(18), 7361-7366.

Shannon, C. E. (1948). A mathematical theory of communication. Bell System Technical Journal, 27(3), 379-423. doi: 10.1002/j.1538-7305.1948.tb01338.x

Smith, S. L., Smith, I. T., Branco, T., \& Häusser, M. (2013). Dendritic spikes enhance stimulus selectivity in cortical neurons in vivo. Nature, 503(7474), 115-120.

Sperber, D., \& Wilson, D. (1986). Relevance: Communication and Cognition. New Jersey: Blackwell Publishers.

Stivers, T. (2010). An overview of the question-response system in American English conversation. Journal of Pragmatics, 42(10), 2772-2781. doi: 
10.1016/j.pragma.2010.04.011

Stolk, A., D’Imperio, D., di Pellegrino, G., \& Toni, I. (2015). Altered communicative decisions following ventromedial prefrontal lesions. Current Biology: CB, 25(11), 14691474.

Stolk, A., Noordzij, M. L., Verhagen, L., Volman, I., Schoffelen, J.M., Oostenveld, R., Hagoort, P., \& Toni, I. (2014). Cerebral coherence between communicators marks the emergence of meaning. Proceedings of the National Academy of Sciences, 111(51), 18183-18188. doi: 10.1073/pnas.1414886111

Stolk, A., Noordzij, M. L., Volman, I., Verhagen, L., Overeem, S., van Elswijk, G., Bloem, B., Hagoort, P., \& Toni, I. (2014). Understanding communicative actions: A repetitive TMS study. Cortex, 51, 25-34. doi: 10.1016/j.cortex.2013.10.005

Stolk, A., Verhagen, L., Schoffelen, J.M., Oostenveld, R., Blokpoel, M., Hagoort, P., van Rooij, I., \& Toni, I. (2013). Neural mechanisms of communicative innovation. Proceedings of the National Academy of Sciences of the United States of America, 110(36), 14574-14579.

Stolk, A., Verhagen, L., \& Toni, I. (2016). Conceptual alignment: How brains achieve mutual understanding. Trends in Cognitive Sciences, 20(3), 180-191.

Tomasello, M., Carpenter, M., Call, J., Behne, T., \& Moll, H. (2005). Understanding and sharing intentions: The origins of cultural cognition. The Behavioral and Brain Sciences, 28(5), 675-691; discussion 691-735.

Toni, I., \& Stolk, A. (2019). Conceptual alignment as a neurocognitive mechanism for human communicative interactions. In P. Hagoort (Ed.), Human language: From genes and brains to behavior, 249. Cambridge, MA: The MIT Press.

Veissière, S. P. L., Constant, A., Ramstead, M. J. D., Friston, K. J., \& Kirmayer, L. J. (2019). Thinking through other minds: A variational approach to cognition and culture. The 
Behavioral and Brain Sciences, 43, e90.

Wadge, H., Brewer, R., Bird, G., Toni, I., \& Stolk, A. (2019). Communicative misalignment in autism spectrum disorder. Cortex; a Journal Devoted to the Study of the Nervous System and Behavior, 115, 15-26.

Warglien, M., \& Gärdenfors, P. (2015). Meaning negotiation. Applications of Conceptual Spaces, 79-94. doi: 10.1007/978-3-319-15021-5_5 